\title{
Seroprevalence of antibodies against SARS-CoV-2 among health care workers in a large Spanish reference hospital
}

\author{
Alberto L. Garcia-Basteiro (10 1,2,3,12凶, Gemma Moncunill (10) 1,12, Marta Tortajada4, Marta Vidal', \\ Caterina Guinovart', Alfons Jiménez 1,5, Rebeca Santano (1) 1, Sergi Sanz 10 1,5,6, Susana Méndez 1,5, \\ Anna Llupià (10) 1,7, Ruth Aguilar (10) 1, Selena Alonso (10 1, Diana Barrios ${ }^{1}$, Carlo Carolis (10 8, Pau Cisteró1, \\ Eugenia Chóliz ${ }^{9}$, Angeline Cruz (10) ${ }^{1}$, Silvia Fochs ${ }^{1}$, Chenjerai Jairoce ${ }^{1,3}$, Jochen Hecht ${ }^{8}$, Montserrat Lamoglia (1) 1,10, \\ Mikel J. Martínez ${ }^{1,11}$, Robert A. Mitchell ${ }^{1}$, Natalia Ortega ${ }^{1}$, Nuria Pey ${ }^{1}$, Laura Puyol ${ }^{1}$, Marta Ribes ${ }^{1}$, Neus Rosell', \\ Patricia Sotomayor', Sara Torres', Sarah Williams', Sonia Barroso4, Anna Vilella (1) 1,7, José Muñoz ${ }^{1,2}$, \\ Antoni Trilla (1) 1,7,9, Pilar Varela4, Alfredo Mayor ${ }^{1,3,5,13}$ \& Carlota Dobaño (D) 1,5,13
}

Health care workers (HCW) are a high-risk population to acquire SARS-CoV-2 infection from patients or other fellow HCW. This study aims at estimating the seroprevalence against SARS-CoV-2 in a random sample of HCW from a large hospital in Spain. Of the 578 participants recruited from 28 March to 9 April 2020, 54 (9.3\%, 95\% Cl: 7.1-12.0) were seropositive for IgM and/or IgG and/or IgA against SARS-CoV-2. The cumulative prevalence of SARS-CoV-2 infection (presence of antibodies or past or current positive rRT-PCR) was $11.2 \%(65 / 578,95 \% \mathrm{Cl}: 8.8-14.1)$. Among those with evidence of past or current infection, $40.0 \%(26 / 65)$ had not been previously diagnosed with COVID-19. Here we report a relatively low seroprevalence of antibodies among HCW at the peak of the COVID-19 epidemic in Spain. A large proportion of HCW with past or present infection had not been previously diagnosed with COVID-19, which calls for active periodic rRT-PCR testing in hospital settings.

\footnotetext{
${ }^{1}$ ISGlobal, Hospital Clínic - Universitat de Barcelona, Barcelona, Spain. ${ }^{2}$ International Health Department, Hospital Clínic, Universitat de Barcelona, Barcelona, Spain. ${ }^{3}$ Centro de Investigação em Saúde de Manhiça (CISM), Maputo, Mozambique. ${ }^{4}$ Occupational Health Department, Hospital Clínic, Universitat de Barcelona, Barcelona, Spain. ${ }^{5}$ Spanish Consortium for Research in Epidemiology and Public Health (CIBERESP), Madrid, Spain. ${ }^{6}$ Department of Basic Clinical Practice, Faculty of Medicine, Universitat de Barcelona, Barcelona, Spain. ${ }^{7}$ Department of Preventive Medicine and Epidemiology, Hospital Clinic, Universitat de Barcelona, Barcelona, Spain. ${ }^{8}$ Centre for Genomic Regulation (CRG), The Barcelona Institute of Science and Technology, Barcelona, Spain. ${ }^{9}$ Faculty of Medicine and Health Sciences, Universitat de Barcelona, Barcelona, Spain. ${ }^{10}$ Faculty of Health Sciences of Blanquerna, Universitat Ramon Llull de Barcelona, Barcelona, Spain. ${ }^{11}$ Department of Microbiology, Hospital Clinic, Universitat de Barcelona, Barcelona, Spain. ${ }^{12}$ These authors contributed equally: Alberto L. Garcia-Basteiro, Gemma Moncunill. ${ }^{13}$ These authors jointly supervised this work: Alfredo Mayor, Carlota Dobaño.

凶email: alberto.garcia-basteiro@isglobal.org
} 
C OVID-19 is a novel viral disease caused by SARS-CoV-2 that was first detected in Wuhan, China, in December $2019^{1}$. Given the alarming levels of spread, severity of disease, and number of affected countries, the World Health Organization (WHO) declared COVID-19 as a pandemic on March 11th, $2020^{2}$. The clinical syndrome caused by SARS-CoV2 ranges from very mild symptomatology to severe pneumonia, acute respiratory distress syndrome, and death ${ }^{3}$. However, several reports show that many individuals might carry the virus without presenting any symptoms for several weeks ${ }^{4-6}$. Thus, the exact number of individuals who have been infected by SARS-CoV-2 is currently unknown.

Health care workers (HCW) are the frontline workforce for clinical care of suspected and confirmed COVID-19 cases. Consequently, they are presumably exposed to a higher risk of acquiring the disease than the general population and, if infected, pose a risk to vulnerable patients and fellow $\mathrm{HCW}^{7}$. In a tertiary hospital in Madrid, Spain (one of the regions with the highest COVID-19 attack rates in the country), 38\% (791/2085) of HCW tested positive for SARS-CoV-2 by real-time reverse-transcriptase polymerase chain reaction (rRT-PCR) in March 2020 (11.6\% of all hospital workers $)^{8}$. HCW with a positive rRT-PCR diagnosis need to be isolated, and their close contacts-many of them coworkers-should be quarantined. Thus, if transmission rises, the number of frontline HCW could become insufficient to respond to the healthcare demand. To cope with this scenario, several strategies, including periodic screenings, weekly-shifts, and other organizational measures are being implemented in a variety of settings to guarantee proper patient care ${ }^{9}$. Nonetheless, quantification and characterization of SARS-CoV-2 infection within health care facilities is unknown in most countries hard-hit by the COVID-19 epidemic.

Seroprevalence studies can provide relevant information on the proportion of people who have experienced a recent or past infection. They are relevant when conducted in the community, but also for critical population subgroups such as nursing homes or health care facilities. Monitoring the prevalence of infection among HCW (regardless of history of symptoms) is useful for assessing the level of exposure among hospital personnel and identifying high-risk departments. Likewise, knowledge of past infection among HCW could be useful for avoiding unnecessary quarantines and for health care resource planning ${ }^{10}$. Although there is a growing body of evidence on the immunological responses against SARS-CoV-2, the time to seroconversion and the antibody levels elicited are not well characterized yet. Importantly, the correlation between seropositivity or antibody levels and protection against reinfection, as well as the duration of protective immunity, remains to be elucidated ${ }^{11}$.

This study aims to estimate the seroprevalence of antibodies against SARS-CoV-2 and characterize the antibody profile in HCW from Hospital Clínic of Barcelona (HCB), one of the reference centers in Spain for the diagnosis and treatment of COVID-19 disease. As a secondary objective, we aim to assess the overall infection prevalence (past and current) to SARS-CoV-2 as well as the prevalence of asymptomatic infections.

\section{Results}

Baseline characteristics. From a total number of 5598 HCW registered at HCB as of March 9th 2020, we approached the first 1172 randomly selected individuals, following the order of the list. Of these, 798 were eligible to participate and 583 were recruited, yielding a participation rate of $74.3 \%$. We then excluded five recruited participants after re-checking inclusion and exclusion criteria (Fig. 1). A total of 578 participants were included in the analysis, of whom 314 (54.3\%) were younger than 45 years of age.

The mean age of participants was 42.1 years (SD: 11.6$)$ and $72.1 \%$ were female. Around half $(288 / 578,49.8 \%)$ were nurses, auxiliary nurses or stretcher-bearers, and $25.4 \%$ (147/578) were physicians. Eleven per cent of the participants reported having comorbidities and $36.3 \%$ reported having had COVID-19-compatible symptoms in the previous months. Thirty-nine participants $(6.7 \%)$ had been previously diagnosed with COVID-19 confirmed by rRT-PCR, of which only one had required hospital admission (Table 1 and Supplementary Table 1).

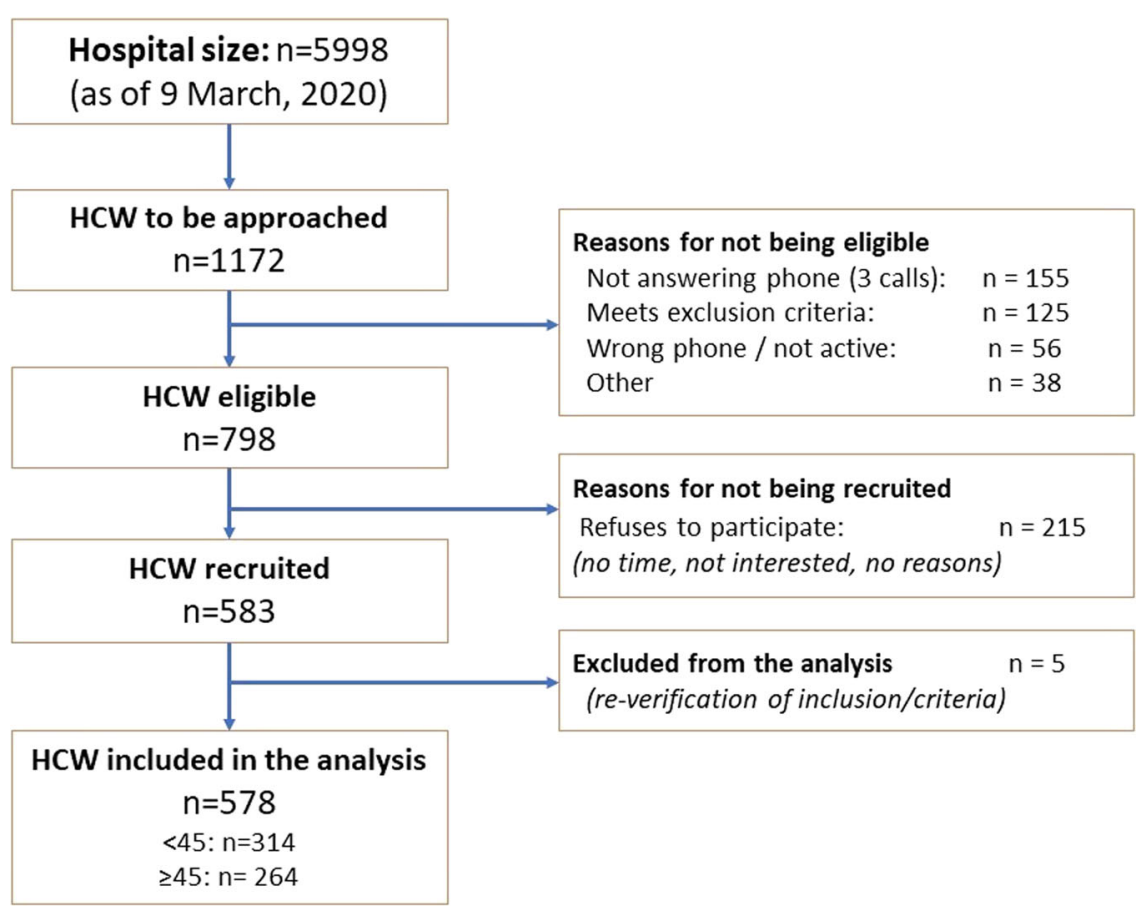

Fig. 1 Study participant flowchart. 
Prevalence of current infection as determined by a positive rRT-PCR. Fifteen participants (2.6\%, 95\% CI: $1.5-4.3 \%)$ had a positive rRT-PCR of SARS-CoV-2 at the time of recruitment and 0.003 had an invalid rT-PCR result. Among participants with a positive rRT-PCR at recruitment, 9 of $15(60.0 \%)$ had a previous COVID-19 diagnosis, and 3 of 15 (20.0\%) did not report any COVID-19-compatible symptom in the previous months. The mean time since diagnosis among those participants with a previous COVID-19 diagnosis and a positive rRT-PCR at recruitment was 9 days (range 2-17). Only 1 of the 6 participants with positive rRT-PCR at recruitment and no history of previous COVID-19 diagnosis had detectable antibodies.

Seroprevalence of antibodies against SARS-CoV-2. Fifty-four participants (9.3\%, 95\% CI: 7.1-12.0) were seropositive for IgM

\begin{tabular}{|c|c|c|}
\hline & & Total \\
\hline \multirow[t]{2}{*}{ Sexa } & Male & $161(28 \%)$ \\
\hline & Female & $417(72 \%)$ \\
\hline \multirow[t]{4}{*}{ Professional category ${ }^{a}$} & $\begin{array}{l}\text { Nurse/Auxiliary } \\
\text { nurse/Stretcher-bearer }\end{array}$ & $288(50 \%)$ \\
\hline & Physician & $147(25 \%)$ \\
\hline & $\begin{array}{l}\text { Lab technologist/other } \\
\text { tech. }\end{array}$ & $45(8 \%)$ \\
\hline & $\begin{array}{l}\text { Administrative officers, } \\
\text { Other }^{b}\end{array}$ & $98(17 \%)$ \\
\hline \multicolumn{2}{|l|}{$\mathrm{Age}^{\mathrm{C}}$} & $42,1(11.6)$ \\
\hline \multirow[t]{2}{*}{ Daily contact with patients ${ }^{a}$} & No & $123(21 \%)$ \\
\hline & Yes & $455(79 \%)$ \\
\hline \multirow[t]{2}{*}{ Working in a COVID-19 unita } & No & $315(54 \%)$ \\
\hline & Yes & $263(46 \%)$ \\
\hline Close contact with confirmed & No & $137(24 \%)$ \\
\hline COVID19 or suspected case ${ }^{a}$ & Yes & $441(76 \%)$ \\
\hline Previously diagnosed with & No & $539(93 \%)$ \\
\hline COVID-19 by rRT-PCR ${ }^{a}$ & Yes & $39(7 \%)$ \\
\hline \multirow[t]{2}{*}{ Comorbidities ${ }^{a}, d$} & No & $517(89 \%)$ \\
\hline & Yes & $61(11 \%)$ \\
\hline & $2.8(1.2)$ \\
\hline \multirow{4}{*}{$\begin{array}{l}\text { Household size } \\
\text { Received Flu vaccine } \\
\text { (2019-2020 season) }^{a} \\
\text { Reporting COVID-19 compatible } \\
\text { symptoms within previous } \\
\text { months }^{a}\end{array}$} & No & $339(59 \%)$ \\
\hline & Yes & $239(41 \%)$ \\
\hline & No & $368(64 \%)$ \\
\hline & Yes & $210(36 \%)$ \\
\hline \multicolumn{3}{|c|}{ 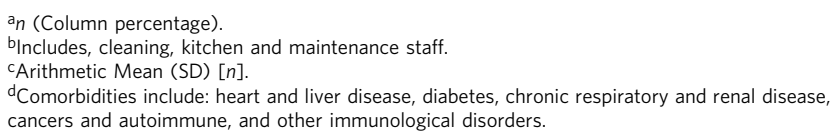 } \\
\hline
\end{tabular}

and/or IgG and/or IgA against SARS-CoV-2 (Table 2). A total of $36(6.2 \%), 44(7.6 \%)$, and $47(8.1 \%)$ participants were positive for IgM, IgG, and IgA, respectively (Fig. 2). Four participants were seropositive for IgM only (IgG- \& IgA-), two were seropositive for IgG only (IgM- \& IgA-), and five were seropositive for IgA only (IgG- \& IgM-). Around 15\% (6/39) of HCW who had been previously diagnosed with COVID-19 by rRT-PCR did not show a detectable response of any of the antibody isotypes (Supplementary Table 2). However, the days since onset of symptoms to recruitment were $<10$ in 4 out of the 6 individuals without detectable antibodies and one individual had no symptoms. Twenty per cent (11/54) of seropositive participants did not report COVID-19 compatible symptoms in the previous months. Around 39\% (21/54) of seropositive HCW had never been diagnosed of COVID-19, although 10 of these (47.6\%) reported past COVID-19-compatible symptoms.

The odds of being seropositive were higher in participants who reported having had any COVID-19-compatible symptom in the previous months (adjusted OR: 8.8, 95\% CI 4.41-17.73, $p<$ 0.0001 ) (Table 3). The individual symptoms more strongly associated with seropositivity were (in order): anosmia (OR: 83.0, 95\% CI: 29.6-232.9), ageusia (OR: 71.4, 95\% CI: 25.4-200.8), fever (OR: 11.4, 95\% CI: 6.0-31.3) and fatigue (OR: 11.2, 95\% CI: 6.1-20.7), all of them with a $p<0.0001$ in the univariable analysis. There was some evidence in the multivariable logistic regression models (MLM) that those with higher household size had higher odds of being seropositive (OR for every additional household member: 1.3 ; 95\% CI: $0.96-1.62 ; p$ value (Wald test) $=0.09$ ). The professional category, working in COVID-19 units, daily contact with patients, close contact with a COVID-19 case, comorbidities or sex, did not show any statistically significant association with presence of antibodies to SARS-CoV-2 (Table 3, Supplementary Fig. 2).

Among seropositive $\mathrm{HCW}$, there were no statistically significant associations of antibody levels with sex (Fig. 3a). IgM levels positively correlated with age (rho $=0.36, p$ value (Spearman $=0.031$ ) (Fig. 3b). IgA levels were higher in participants reporting COVID-19-compatible symptoms in the previous months than in those reporting being asymptomatic $(p=0.041)$ (Fig. 3c), and among symptomatic individuals, duration of symptoms $>10$ days was associated with higher IgM levels $(p=$ 0.022) (Fig. 3d).

Among HCW reporting symptoms in the last months, antibodies were detected in individuals with 6 or more days between symptoms onset and recruitment for $\operatorname{IgA}$ and later for IgM and IgG (Fig. 4), with no seropositive results detected among individuals with symptoms onset $<6$ days prior to the recruitment visit. In fact, we only detected antibodies in two participants surveyed $<10$ days after onset of symptoms (Supplementary Table 3). Antibody levels increased and peaked between day 20

Table 2 Overall proportion of HCW with (a) detectable antibodies, (b) history of past positive rRT-PCR, (c) Positive rRT-PCR at study recruitment, and (d) Cumulative prevalence of infection (past/current rRT-PCR and/or antibodies).

\begin{tabular}{|c|c|c|c|c|c|}
\hline & $n$ & Total & $\%(95 \% \mathrm{Cl})$ & $\begin{array}{l}\text { Not previously diagnosed as COVID-19 } \\
\text { by rRT-PCR }(n(\%))\end{array}$ & 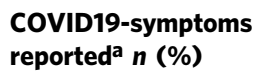 \\
\hline $\begin{array}{l}\text { Seropositive to SARS CoV-2 Antibodies (IgA } \\
\text { and/or IgM and/or lgG) }\end{array}$ & 54 & 578 & $9.3 \%(7.1-12.0)$ & $21(38.9 \%)$ & $10(47.6 \%)$ \\
\hline Positive rRT-PCR at study recruitment ${ }^{b}$ & 15 & 576 & $2.6 \%(1.5-4.3)$ & $6(42.9 \%)$ & $3(50.0 \%)$ \\
\hline $\begin{array}{l}\text { Any evidence of past/current infection by } \\
\text { rRT-PCR of serology }\end{array}$ & 65 & 578 & $11.2 \%(8.8-14.1)$ & $26(40.0 \%)$ & $12(46.2 \%)$ \\
\hline
\end{tabular}




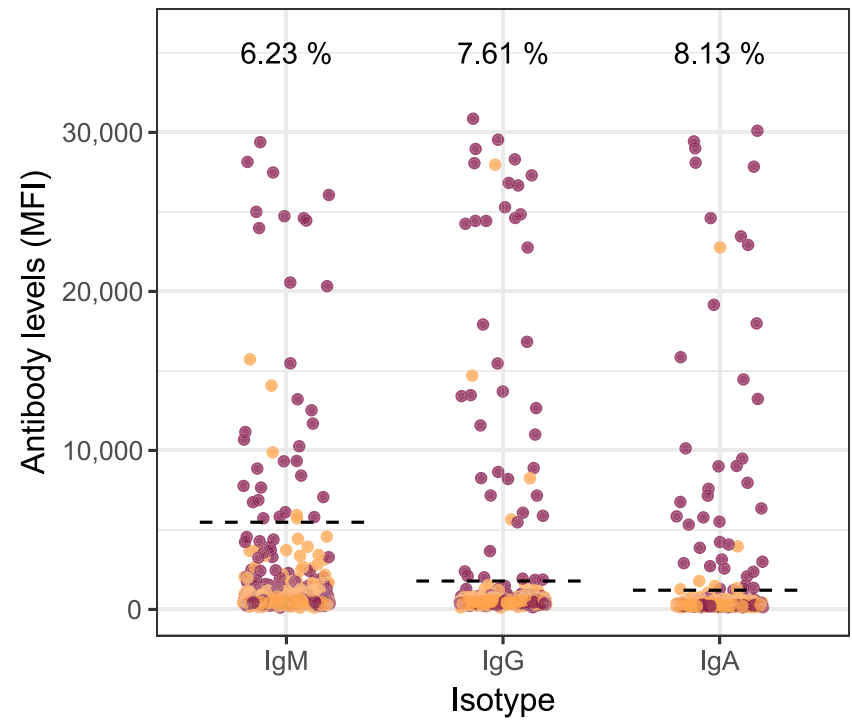

Symptoms - No $\bullet$ Yes

Fig. 2 SARS-CoV-2 antibody levels in all study participants. Dots depict the levels (median fluorescence intensity, MFI) of $\operatorname{lgM}$, IgG, and IgA against Receptor Binding Domain (RBD) of the SARS-CoV-2 Spike glycoprotein. Dashed lines indicate the seropositivity threshold calculated with prepandemic controls as the 10 to the mean plus 3 standard deviations of $\log _{10}$-transformed MFIs. The percentage of seropositive subjects is shown for each antibody isotype. Orange and burgundy dots show subjects who did not have or did have history of at least one COVID-19 compatible symptom, respectively. $N=578$.

and 25 for IgM and IgG, and a few days earlier for IgA. There were only three seropositive $\mathrm{HCW}$ with symptoms onset having occurred earlier than 25 days prior to survey (Supplementary Table 3).

Overall cumulative prevalence of past or current infection. Sixty-five HCW had either a positive rRT-PCR in the past or at survey recruitment, or had a positive antibody response (Table 2). Thus, the cumulative prevalence of SARS-CoV-2 infection was 11.2\% (95\% CI: 8.8-14.1). Among them, 23.1\% (15/65) did not report any COVID-19 compatible symptom in the previous months. Forty per cent (26/65) had not been previously diagnosed with COVID-19, although 12 of them reported COVID-19 compatible symptoms.

\section{Discussion}

This is, to our knowledge, the first study reporting seroprevalence of antibodies against SARS-CoV-2 among a representative sample of HCW in a COVID-19 high burden country. We found that 9.3\% (95\% CI: 7.2-12.0) of HCW from a large Spanish referral hospital (recruited from March 28th to April 9th, 2020) developed detectable IgA, IgG, and/or IgM antibodies. Given that $\mathrm{HCW}$ are a high-risk population for SARS-CoV-2, it is likely that the community seroprevalence is lower than this figure, showing that we are still very far from reaching the $67 \%$ herd immunity level that is estimated to be needed to protect the susceptible population ${ }^{12}$, assuming that this immunity prevents from reinfection. The seroprevalence found was lower than expected, based on the large number of rRT-PCR positive cases reported in a referral hospital in Madrid in March 2020 (11.6\% of all hospital workers $)^{8}$, and an estimate from modeling studies of $15 \%$ seroprevalence for the overall Spanish population in March $2020^{13}$. However, it is compatible with the $7.1 \%$ (95\% CI: 5.9-8.5\%) preliminary seroprevalence results for Barcelona province, as reported by the national Ministry of Science in the period 27 April-11 May 202014. The likely higher availability of PPE compared with other hospitals, and the early implementation of rRT-PCR screening programs in HCW working in COVID-19 units, coupled with timely case identification and effective contact tracing and quarantines for those outside COVID-19 unit, could explain a relatively low number of infections in our study.

Combining data from antibody detection and previous or current positive rRT-PCR, the cumulative prevalence of SARSCoV-2 infection rose to $11.2 \%$. However, $40.0 \%$ of the seropositive HCW had not previously been diagnosed with COVID19 and $23.1 \%$ were asymptomatic, indicating a large percentage of infections were undetected $^{15}$. This calls for early detection/ screening programs to be broadly and timely implemented in HCW to decrease in-hospital transmission as well as reinforce the critical role of PPE usage ${ }^{16}$.

The likelihood of being seropositive was higher in participants who reported having had any COVID-19 symptom within the last months (OR: 8.84 ) and $80 \%$ of seropositive HCW did report having had symptoms. Although most COVID-19 symptoms are common to many other upper respiratory viral infections, those more highly associated with seropositivity were by far anosmia and ageusia (both OR $>70$ ) that, although infrequent, seem to be quite specific for COVID-1917,18. As expected, having developed the disease was the most important factor associated with the development of antibodies (OR: 135.6). In addition, there was some evidence that the higher the size of the household, the higher the odds (OR: 1.25$)$ of being seropositive $(p<0.09)$, potentially because household exposure is an added source of infection among HCW. None of the professional categories or being directly involved in clinical care were factors associated with higher odds of being seropositive. Working in a COVID-19 unit was also not associated with seropositivity, which might be explained by a higher perception of risk leading to a better protection with PPEs, more careful practices and thus, a lower risk of acquiring the infection ${ }^{19}$. Nonetheless, the relatively low number of seropositive HCW in our sample hinders any firm conclusion about associations between professional categories, level of patient interaction, and risk of infection.

Using only the RBD antigen in the assay but three different isotypes, we could detect antibodies in $97 \%$ of participants with a previous positive rRT-PCR and more than 10 days since onset of symptoms. This is in line with previous reports showing that seroconversion occurs between 2-3 weeks after onset of symptoms ${ }^{11}$. Importantly, we detected lower IgA levels in seropositive participants without symptoms, in line with a previous observation of correlation of IgA levels and COVID-19 severity (preprint publication $)^{20}$. If it is confirmed that asymptomatic subjects have lower levels of antibodies ${ }^{21}$, this could impact detection of seroconversion in this specific group. We cannot discard that some participants may be either very low or non-responders, as several reports have found COVID-19 patients with low or no responses for IgM, IgG, or neutralizing antibodies ${ }^{22,23}$.

By increasing the number of viral antigens in our assay we may allow to increase its sensitivity, as responses to different antigens may present different kinetics and vary between individuals 24,25 . Nonetheless, we included determinations to three isotypes to capture a variety of responses between individuals and their relation to time from onset of symptoms. Also, their maintenance and role in protection are probably different. IgM is the first antibody being produced by $\mathrm{B}$ cells upon antigenic 


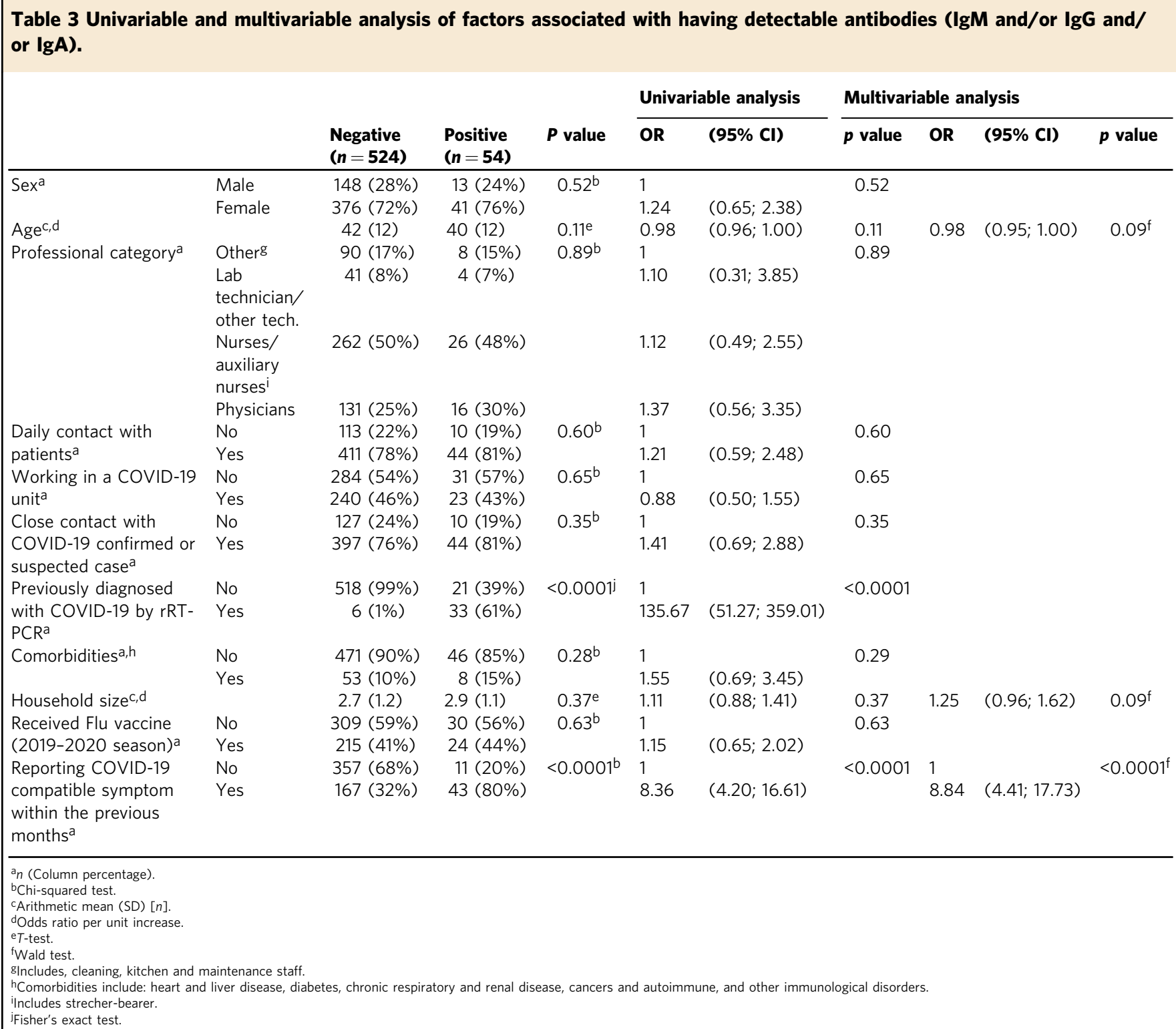

encounter, IgA is key for mucosal immunity, and IgG is considered to be the most important for memory responses, but their respective kinetics will only be well characterized over a longitudinal follow-up study. We found few participants with IgA only, IgM only, or IgG only, and no evidence that specific antibody profiles are associated with the onset of symptoms or current positive rRT-PCR. Therefore, our data do not support that antibody responses could contribute to diagnosis of acute infection (IgM detection \pm increasing IgG) versus past infection (negative or low IgM and persisting IgG) as previously suggested $^{26}$. However, our analysis of antibody levels and seroprevalence by days since onset of symptoms suggests that $\operatorname{IgA}$ responses can be detected and peak earlier than IgM and IgG, consistent with previous reports ${ }^{27}$.

This study has several limitations. First, we collected data over a 12-day period, which, in the context of a rapidly growing epidemic, hinders its association to a specific date, with the prevalence having to be interpreted as the average prevalence over those 12 days. Second, we only collected nasopharyngeal samples (instead of oropharyngeal and nasopharyngeal) from study participants for the molecular detection of SARS-CoV-2 RNA. Although this could reduce rRT-PCR sensitivity, there is evidence showing that nasopharyngeal samples have a higher positivity rate than oropharyngeal samples ${ }^{28}$. Third, seroprevalence was defined as positivity of any of the antibody isotypes (IgM, IgG, and $\operatorname{IgA}$ ), which maximized sensitivity rather than specificity. However, our Luminex assay validation showed excellent specificity for the three isotypes, thus, our potential overestimation of the true prevalence is likely to be minimized. Finally, our participation rate $(74 \%)$ could have introduced selection bias in our sample. It could be that many of those refusing to participate might have had a characteristic associated to an increased risk of infection (being very busy at COVID-19 units, for example). Thus, the impact is potentially minimal, given the lack of association of this and most studied variables with our primary endpoint.

In conclusion, the seroprevalence of antibodies to SARS-CoV-2 was lower than expected. Most participants with a confirmed COVID-19 diagnosis elicited antibody responses (IgA, IgG and/ or $\operatorname{IgM})$, with $\operatorname{IgA}$ demonstrating the highest sensitivity in the 

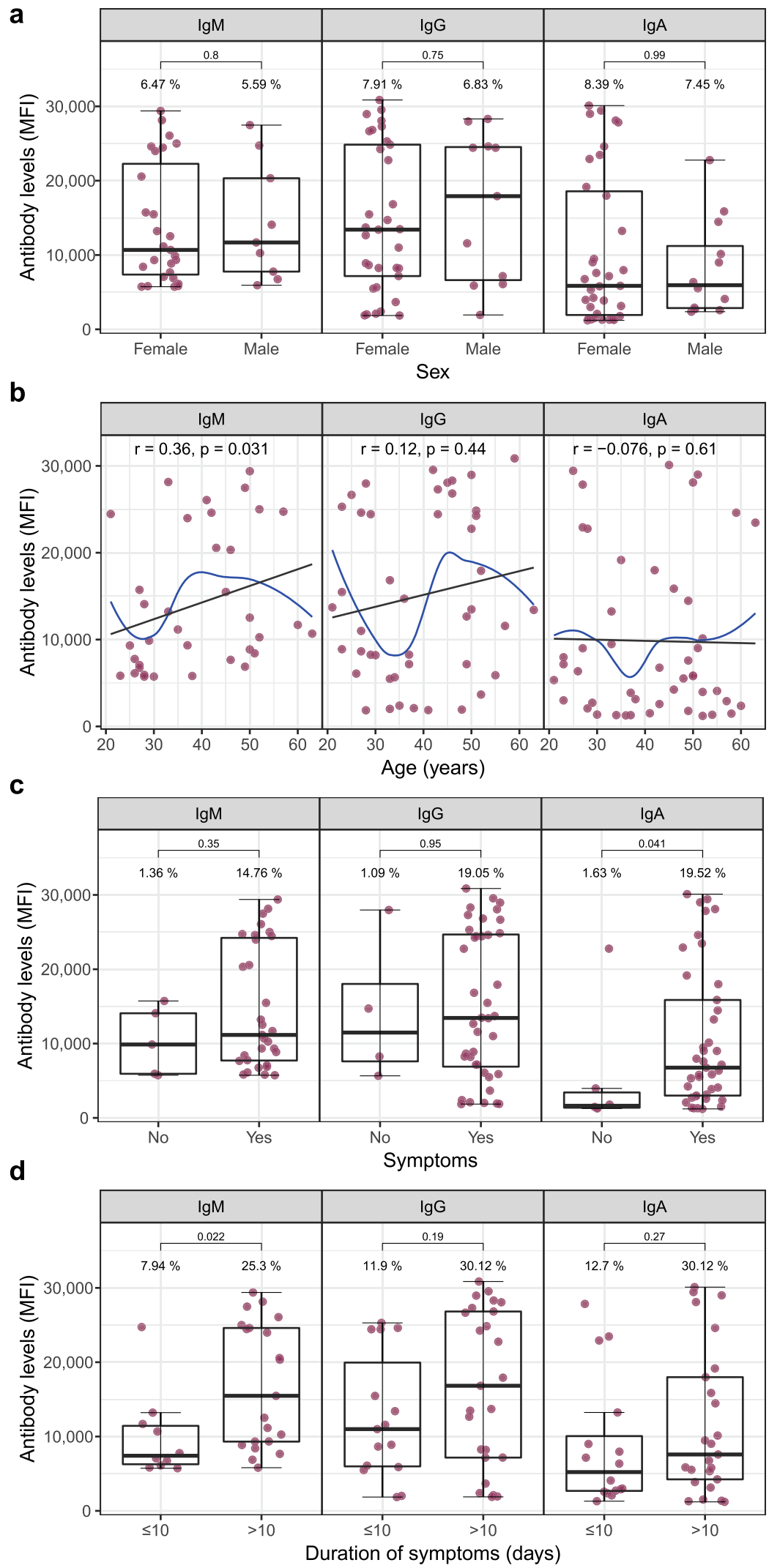

initial days after symptoms onset. Given the current lack of evidence on the correlation of SARS-CoV-2 antibody levels and protection against reinfection, no recommendations should be derived for seropositive HCW at an individual level. This study also shows that around $46 \%$ of undiagnosed infections occur in
HCW who report having experienced COVID-19 compatible symptoms. Thus, enforcement of rRT-PCR screening programs for all HCW, regardless of the presence of symptoms, is highly recommended in healthcare settings to reduce the risk of hospital-acquired SARS-CoV-2 infections. 
Fig. 3 SARS-CoV-2 antibody levels by demographic and clinical variables. Levels (median fluorescence intensity, MFI) of IgM, IgG, and IgA against Receptor Binding Domain (RBD) of the SARS-CoV-2 Spike glycoprotein by sex (a), age (b), symptoms (c), and duration of symptoms (d). For (a-c), data are shown only for seropositive subjects for $\lg M(N=36)$, for $\lg (N=44)$, and for $\lg A(N=47)$. For $(\mathbf{d})$, data are shown only for seropositive and symptomatic subjects for $\lg M(N=31)$, for $\lg G(N=40)$, and for $\lg A(N=41)$. Percentages indicate the proportion of seropositive subjects within each category of the x-axis. The center line of boxes depicts the median of MFIs; the lower and upper hinges correspond to the first and third quartiles; the distance between the first and third quartiles corresponds to the interquartile range (IQR); whiskers extend from the hinge to the highest or lowest value within $1.5 \times$ IQR of the respective hinge. Wilcoxon rank test was used to assess statistically significant differences in antibody levels between groups in (a, $\mathbf{c}$ and $\mathbf{d}$ ). Spearman test was used to calculate the correlation coefficients $(r)$ and $p$ values $(p)$ in $(\mathbf{b})$, where the black line depicts linear regression and the blue curve represents nonlinear regression calculated using the LOESS (locally estimated scatterplot smoothing) method.
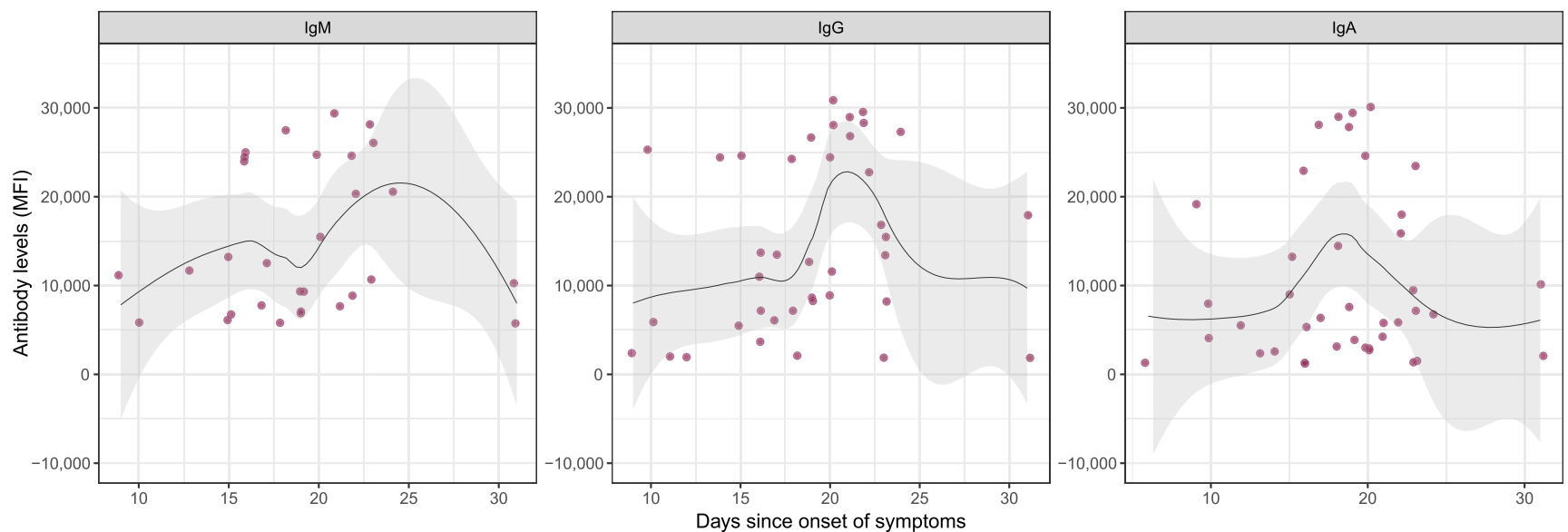

Fig. 4 SARS-CoV-2 antibody levels by time since onset of symptoms in seropositive subjects. Levels (median fluorescence intensity, MFI) of IgM, IgG, and IgA against Receptor Binding Domain of the SARS-CoV-2 Spike glycoprotein by days since onset of any symptom. Data are shown only for seropositive subjects with any symptom compatible with COVID-19 ( $n=30$ for $\operatorname{lgM}, 39$ for $\lg$, and 40 for $\lg A$ ). The fitting curve was calculated using the LOESS (locally estimated scatterplot smoothing) method. Shaded areas represent $95 \%$ confident intervals. One subject seropositive for the three isotypes and who started symptoms 40 days before serological testing is not shown.

\section{Methods}

Study design, population and setting. The study design consists of four crosssectional surveys (at baseline, 1 month, 6 months and 12 months) in the same cohort of randomly selected HCW from HCB. We hereby present the first crosssectional survey, conducted from March 28th to April 9th, 2020. The study population was defined as those who deliver care and services to patients, either directly as physicians or nurses, or indirectly as assistants, technicians, stretcherbearers, or other support staff (administrative officers, cleaning, kitchen, laundry, maintenance, etc. $)^{29}$. Inclusion criteria included being an adult ( $>17$ years) worker at HCB registered at the Human Resources department. Exclusion criteria included: (a) absenteeism from workplace in the last 30 days (i.e., on vacation, sick leave, sabbatical), (b) working exclusively outside the HCB or Maternity main buildings with no interaction with patients on a daily basis, (c) retirement or end-of-contract planned within one year after the recruitment date, and (d) participating in COVID-19 clinical trials for preventive or treatment therapies.

HCB is a large University of Barcelona teaching hospital. With over 700 beds, it is the main public supplier of specialized health services for a population of around 540,000 inhabitants and also acts as a tertiary referral hospital ${ }^{30}$.

Procedures. A random sample of HCW was selected from the HCB's Human Resources database (as of March 9th, 2020). Selected individuals were approached telephonically following the order of the random list were excluded upon review of inclusion and exclusion criteria or after three phone calls (different days) without response.

After obtaining written informed consent, we filled out a standardized electronic questionnaire programmed in REDCap (Research Electronic Data Capture $)^{31}$ for each participant, with the following information: demographics (age, sex, household size, etc.), professional information (occupation, hospital department, and shift), clinical information such as history of COVID-19compatible symptoms during the previous months (cough, sore throat, runny nose, fatigue, shortness of breath, fever, headache, vomiting, diarrhea, anosmia, ageusia, and chills) date of onset and resolution of symptoms, history of rRT-PCR testing, comorbidities, and history of close contact with COVID-19 cases.

We collected a nasopharyngeal swab (DeltaLabs ref: 304273) for the detection of SARS-CoV-2 RNA by rRT-PCR and a venous blood draw for immunological assessments. Both procedures were performed by trained nurses using appropriate personal protective equipment (PPE). Samples were transported to the laboratory within $3 \mathrm{~h}$ of sample collection. Nasopharyngeal swabs and plasma samples were stored at $-80^{\circ} \mathrm{C}$ until analysis.

For participants reporting to be isolated at home (i.e., due to a COVID-19 diagnosis) or on quarantine, data, and specimen collection took place at their households following the relevant biosafety protocols.

Laboratory procedures. $r R T$-PCR. After adding $500 \mu \mathrm{l}$ of Zymo DNA/RNA Shield Lysis Buffer to the same amount of nasopharyngeal sample collection media, RNA was extracted using the Quick-DNA/RNA Viral MagBead kit (Zymo) and the TECAN Dreamprep robot. Five microliters of RNA solution were added to $15 \mu \mathrm{l}$ of rRT-PCR master mix (Luna Universal Probe One-Step RT-qPCR Kit; New England Biolabs) and used for amplification of SARS-CoV-2 N1 and N2 regions, as well as the human RNase $\mathrm{P}$ gene as control, using probes, primers and cycling conditions described in the CDC-006-00019 CDC/DDID/NCIRD/ Division of Viral Diseases protocol (3/30/2020 release, Supplementary Note 1). Each batch of RNA extractions and rRT-PCR reactions included three positive controls (EURM019 single stranded RNA fragments of SARS-CoV-2 provided by the European Commission Joint Research Centre), 2019-nCoV_N_Positive Control (IDT integrated technologies, ref. 10006625) and Hs_RPP30 Positive Control (IDT integrated technologies, ref. 10006626), as well as negative controls. A positive result was considered if the Ct values for N1, N2 and RNase P were below 40. Samples discordant for $\mathrm{N} 1$ and $\mathrm{N} 2$ were repeated and samples with a $\mathrm{Ct} \geq 40$ for RNase $\mathrm{P}$ were considered as invalid.

Quantification of antibodies to SARS-CoV-2 by Luminex. To establish seroprevalence, we used a serological assay based on the Luminex technique that has the benefit of a higher dynamic range than other assays, favoring the quantification of immunoglobulin levels. We measured antibodies against the Receptor Binding Domain (RBD) of the spike glycoprotein of SARS-CoV- $2^{32}$, which is, together with the nucleocapsid protein (NP), one of the most immunogenic antigens. Antibodies to RBD correlate with neutralizing antibodies ${ }^{33,34}$ that could be associated with protection based on studies of other coronaviruses and animal models ${ }^{34-37}$. The RBD antigen, kindly donated by the Krammer lab (Mount Sinai, New York) ${ }^{38}$, was coupled to magnetic MAGPLEX $6.5 \mu \mathrm{m} \mathrm{COOH}$-microspheres from Luminex Corporation (Austin, TX) at a concentration of $40 \mu \mathrm{g} / \mathrm{ml}$ for 10,000 beads $/ \mu \mathrm{l}^{39}$.

Antigen-coupled beads were added to a 96 -well $\mu$ Clear ${ }^{\oplus}$ flat bottom plate (Greiner Bio-One, 655096) at 2000 beads/well in a volume of $90 \mu \mathrm{L} /$ well of phosphate buffered saline $+1 \%$ bovine serum albumin $+0.05 \%$ sodium azide 
(PBS-BN). Next, $10 \mu \mathrm{l}$ of test plasma samples (final dilution 1/500), $10 \mu \mathrm{l}$ of a positive control (pool of 20 plasmas from subjects with a positive SARS-CoV-2 rRT-PCR, at four dilutions, $1 / 500,1 / 2000,1 / 8000$ and $1 / 32000$, for QA/QC), and $10 \mu \mathrm{l}$ of two negative controls (plasmas from European subjects collected before the COVID-19 pandemic, at 1/500), were added per plate. Two blank control wells with beads in PBS-BN were set up to measure background signal. Plates were incubated at room temperature (RT) for $2 \mathrm{~h}$ on a microplate shaker at $500 \mathrm{rpm}$ and protected from light. Plates were washed three times with $300 \mu \mathrm{l} /$ well of PBSTween $200.05 \%$, using a magnetic manual washer (Millipore, 43-285). A hundred microliters of biotinylated secondary antibody diluted in PBS-BN (anti-human IgG, B1140, 1/1250; anti-human IgM, B1265, 1/1000; or anti-human IgA, SAB3701227, 1/500; Sigma) were added to all wells and incubated for $45 \mathrm{~min}$ at $500 \mathrm{rpm}$ at RT and protected from light. Plates were washed three times and $100 \mu \mathrm{L}$ of streptavidin-R-phycoerythrin (Sigma, 42250) diluted 1:1000 in PBS-BN were added and incubated during $30 \mathrm{~min}$ at $500 \mathrm{rpm}$, RT and protected from light. Plates were washed three times, and beads resuspended in $100 \mu \mathrm{l}$ of PBS-BN and kept overnight at $4{ }^{\circ} \mathrm{C}$, protected from light. The next day, plates were read using a Luminex xMAP ${ }^{\star} 100 / 200$ analyzer with $70 \mu$ of acquisition volume per well, DD gate 5000-25000 settings, and high PMT option. At least 50 beads were acquired per sample. Crude median fluorescent intensities (MFI) were exported using the xPONENT software. Assay cutoff was calculated as 10 to the mean plus 3 standard deviations of $\log _{10}$-transformed MFIs of 47 negative controls. Sensitivity of the assay using samples from participants previously diagnosed with COVID-19 and with more than 10 days since the onset of symptoms was $97 \%$ for IgA and IgG and $75 \%$ for IgM, with specificities of $100 \%$ for IgG and IgM and $98 \%$ for IgA (Supplementary table 4). The area under the receiver operating characteristic curve (AUC) was $>0.97$ for each of the isotypes using these same samples (Supplementary Fig. 1a) and $>0.87$ using samples from any participant previously diagnosed with COVID-19 regardless of the time since onset of symptoms (Supplementary Fig. 1b).

Sample size and statistical analysis. In order to assess the seroprevalence against SARS-CoV-2 at two time points (month 0 and month 1), with a precision of $5 \%$ and a $95 \% \mathrm{CI}$, a loss to follow up between month 0 and month 1 of $5 \%$ and assuming that the prevalence at month 0 was $30 \%$ and at month 1 was $50 \%$, with a finite population, we estimated we would need $570 \mathrm{HCW}$. Given the uncertainty about what the seroprevalence would be at month 1 , we used $50 \%$, which provides the most conservative sample size.

Seroprevalence of antibodies against SARS-CoV-2, prevalence of SARS-CoV-2 infection by rRT-PCR, and cumulative prevalence of past or current infection (positive SARS-CoV-2 rRT-PCR and/or antibody seropositivity), were calculated as proportions with $95 \%$ CI. We tested the association between variables with the Chisquare or Fisher's exact test (for categorical variables) and $T$ Student test (for continuous quantitative variables). Univariable and MLM were run to evaluate factors associated with seroprevalence of antibodies against SARS-CoV-2. For the variables to be included in the MLM model, we used a stepwise selection, starting with the full model, and using a $p$ value of 0.10 for removal and 0.05 for addition of variables. A diagnosis of COVID-19 was excluded from the MLM because it was assumed to be the source for antibody generation and the high expected correlation with COVID-19 symptoms reported.

Spearman correlations were performed to assess the association of antibody levels with age. Wilcoxon Sum Rank test was used to compare the antibody levels between different groups. Receiver Operating Characteristic (ROC) curves and their correspondent AUC were calculated using the predicted values estimated by logistic regression models with MFI for IgM, IgG, IgA or their combination as predictors and the rRT-PCR result as outcome. The analysis was carried out using the statistical software Stata v16.1 (College Station, TX: StataCorp LLC) and R studio version R-3.5.1 (packages used: ggplot2 and pROC).

Ethical considerations. We have complied with all relevant ethical regulations. The protocol and informed consent form were reviewed and approved by the Institutional Review Board (IRB) at HCB, (CEIm) prior to study implementation (Ref number: $\mathrm{HCB} / 2020 / 0336$ ).

Reporting summary. Further information on research design is available in the Nature Research Reporting Summary linked to this article.

\section{Data availability}

Anonymized data used for this analysis is available and made public under the title of this publication at http://diposit.ub.edu/dspace/handle/2445/56611.

\section{Code availability}

Code used in the analysis is available at http://diposit.ub.edu/dspace/handle/2445/56611.

Received: 28 May 2020; Accepted: 19 June 2020;

Published online: 08 July 2020

\section{References}

1. Zhu, N. et al. A novel coronavirus from patients with pneumonia in China, 2019. N. Engl. J. Med. 382, 727-733 (2020).

2. World Health Organization. WHO Director-General's opening remarks at the media briefing on COVID-19-. https://www.who.int/dg/speeches/detail/whodirector-general-s-opening-remarks-at-the-media-briefing-on-covid-19---11march-2020 (2020).

3. Guan, W. et al. Clinical characteristics of coronavirus disease 2019 in China. N. Engl. J. Med. https://doi.org/10.1056/nejmoa2002032 (2020).

4. Day, M. Covid-19: four fifths of cases are asymptomatic, China figures indicate. $B M J \mathrm{~m} 1375$. https://doi.org/10.1136/bmj.m1375 (2020).

5. Sutton, D., Fuchs, K., D'Alton, M. \& Goffman, D. Universal screening for SARS-CoV-2 in women admitted for delivery. N. Engl. J. Med. https://doi.org/ 10.1056/NEJMc2009316 (2020).

6. Mizumoto, K., Kagaya, K., Zarebski, A. \& Chowell, G. Estimating the asymptomatic proportion of coronavirus disease 2019 (COVID-19) cases on board the Diamond Princess cruise ship, Yokohama, Japan, 2020. Eurosurveillance 25, 2000180 (2020).

7. Ng, K. et al. COVID-19 and the risk to health care workers: a case report. Ann. Intern. Med. https://doi.org/10.7326/L20-0175 (2020).

8. Folgueira, M. D., Munoz-Ruiperez, C., Alonso-Lopez, M. A. \& Delgado, R. SARS-CoV-2 infection in Health Care Workers in a large public hospital in Madrid, Spain, during March 2020. medRxiv 2020.04.07.20055723. https://doi. org/10.1101/2020.04.07.20055723 (2020).

9. Chen, C. \& Zhao, B. Makeshift hospitals for COVID-19 patients: where health-care workers and patients need sufficient ventilation for more protection. J. Hosp. Infect. https://doi.org/10.1016/j.jhin.2020.03.008 (2020).

10. Black, J. R. M., Bailey, C. \& Swanton, C. COVID-19: the case for health-care worker screening to prevent hospital transmission. Lancet (London, England). https://doi.org/10.1016/S0140-6736(20)30917-X (2020).

11. Huang, A. T. et al. A systematic review of antibody mediated immunity to coronaviruses: antibody kinetics, correlates of protection, and association of antibody responses with severity of disease. medRxiv. 2020;2020.04.14.20065771. https://doi.org/10.1101/2020.04.14.20065771 (2020).

12. Kwok, K. O., Lai, F., Wei, W. I., Wong, S. Y. S. \& Tang, J. Herd immunity estimating the level required to halt the COVID-19 epidemics in affected countries. J. Infect. https://doi.org/10.1016/j.jinf.2020.03.027 (2020).

13. Flaxman, S. et al. Report 13: estimating the number of infections and the impact of non-pharmaceutical interventions on COVID-19 in 11 European countries. Imperial College London. https://www.imperial.ac.uk/mrc-globalinfectious-disease-analysis/covid-19/report-13-europe-npi-impact/. Accessed 2nd July 2020.

14. Ministry of Science and Innovation. ESTUDIO ENE-COVID19: PRIMERA RONDA ESTUDIO NACIONAL DE SERO-EPIDEMIOLOGÍA DE LA INFECCIÓN POR SARS-COV-2 EN ESPAÑA. https://www.mscbs.gob.es/ ciudadanos/ene-covid/docs/ESTUDIO_ENE-COVID19_PRIMERA_RONDA_ INFORME_PRELIMINAR.pdf (2020). Accessed 2nd July 2020.

15. Tian, S. et al. Characteristics of COVID-19 infection in Beijing. J. Infect. 80, 401-406 (2020).

16. Htun, H. L. et al. Responding to the COVID-19 outbreak in Singapore: staff protection and staff temperature and sickness surveillance systems. Clin. Infect. Dis. https://doi.org/10.1093/cid/ciaa468 (2020).

17. Vetter, P. et al. Clinical features of covid-19. BMJ 369, m1470 (2020).

18. Pan, L. et al. Clinical characteristics of COVID-19 patients with digestive symptoms in Hubei, China. Am. J. Gastroenterol. 1. https://doi.org/10.14309/ ajg.0000000000000620 (2020).

19. Houghton, C. et al. Barriers and facilitators to healthcare workers' adherence with infection prevention and control (IPC) guidelines for respiratory infectious diseases: a rapid qualitative evidence synthesis. Cochrane Database Syst. Rev. 4, CD013582 (2020).

20. Ma, H. et al. COVID-19 diagnosis and study of serum SARS-CoV-2 specific $\operatorname{IgA}, \operatorname{IgM}$ and $\operatorname{IgG}$ by a quantitative and sensitive immunoassay. medRxiv 2020.04.17.20064907. https://doi.org/10.1101/2020.04.17.20064907 (2020).

21. Wilder-Smith, A. et al. Asymptomatic SARS coronavirus infection among healthcare workers, Singapore. Emerg. Infect. Dis. 11, 1142-1145 (2005).

22. Tan, W. et al. Viral kinetics and antibody responses in patients with COVID-19. medRxiv 2020.03.24.20042382. https://doi.org/10.1101/2020.03.24.20042382 (2020).

23. Wu, F. et al. Neutralizing antibody responses to SARS-CoV-2 in a COVID-19 recovered patient cohort and their implications. medRxiv 2020.03.30.20047365. https://doi.org/10.1101/2020.03.30.20047365 (2020).

24. Dobaño C., et al. Highly sensitive and specific multiplex antibody assays to quantify immunoglobulins $\mathrm{M}, \mathrm{A}$ and $\mathrm{G}$ against SARS-CoV-2 antigens. bioRxiv 2020.06.11.147363; https://doi.org/10.1101/2020.06.11.1 (2020).

25. Burbelo, P. D. et al. Sensitivity in detection of antibodies to nucleocapsid and spike proteins of severe acute respiratory syndrome coronavirus 2 in patients with coronavirus disease 2019. J. Infect. Dis. https://doi.org/10.1093/infdis/ jiaa273 In Press (2020). 
26. Li, Z. et al. Development and clinical application of A Rapid IgM-IgG combined antibody test for SARS-CoV-2 infection diagnosis. J. Med. Virol. https://doi.org/10.1002/jmv.25727 (2020).

27. Hsueh, P. R., Huang, L. M., Chen, P. J., Kao, C. L. \& Yang, P. C. Chronological evolution of IgM, IgA, IgG and neutralisation antibodies after infection with SARS-associated coronavirus. Clin. Microbiol. Infect. 10, 1062-1066 (2004).

28. Wang, X. et al. Comparison of nasopharyngeal and oropharyngeal swabs for SARS-CoV-2 detection in 353 patients received tests with both specimens simultaneously. Int. J. Infect. Dis. https://doi.org/10.1016/j.ijid.2020.04.023 (2020).

29. Mohanty, A., Kabi, A. \& Mohanty, A. P. Health problems in healthcare workers: a review. J. Fam. Med. Prim. Care 8, 2568-2572 (2019).

30. Hospital Clinic de Barcelona. About the Clinic. https://www.clinicbarcelona. org/en/assistance/about-clinic. Accessed 2nd July 2020.

31. Harris, P. A. et al. Research electronic data capture (REDCap)-A metadatadriven methodology and workflow process for providing translational research informatics support. J. Biomed. Inform. 42, 377-381 (2009).

32. Ma, H. et al. Serum IgA, IgM, and IgG responses in COVID-19. Cell. Mol. Immunol. 1-3. https://doi.org/10.1038/s41423-020-0474-z (2020).

33. Berry, J. D. et al. Neutralizing epitopes of the SARS-CoV S-protein cluster independent of repertoire, antigen structure or mAb technology. MAbs 2 53-66 (2010)

34. Jiang, S., Hillyer, C. \& Du, L. Neutralizing Antibodies against SARS-CoV-2 and Other Human Coronaviruses. Trends Immunol. https://doi.org/10.1016/j. it.2020.03.007 (2020)

35. Callow, K. A., Parry, H. F., Sergeant, M. \& Tyrrell, D. A. J. The time course of the immune response to experimental coronavirus infection of man. Epidemiol. Infect. 105, 435-446 (1990).

36. Bao, L. et al. Reinfection could not occur in SARS-CoV-2 infected rhesus macaques. bioRxiv 2020.03.13.990226. https://doi.org/10.1101/2020.03.13.990226 (2020).

37. Liu, W. et al. Two-year prospective study of the humoral immune response of patients with severe acute respiratory syndrome. J. Infect. Dis. 193, 792-795 (2006).

38. Stadlbauer, D. et al. SARS-CoV-2 seroconversion in humans: a detailed protocol for a serological assay, antigen production, and test setup. Curr. Protoc. Microbiol 57, e100 (2020).

39. Vidal, M., Aguilar, R., Campo, J. J. \& Dobaño, C. Development of quantitative suspension array assays for six immunoglobulin isotypes and subclasses to multiple Plasmodium falciparum antigens. J. Immunol. Methods 455, 41-54 (2018).

\section{Acknowledgements}

We would like to thank all the HCW at HCB for their incredible work, endless dedication to patients and resilience. They have given their hearts and health (literally) for people's health. Special thanks to those HCW who participated in this study, for contributing to expanding our knowledge about COVID-19 immunity and epidemiology. Special thanks to ISGlobal colleagues (Antoni Plasencia, Denise Naniche, Gonzalo Vicente, Martine Vrijheid, Matiana González, María Tusell, Cristina Castellana, fellow researchers and administrative department), scientists from Centre for Genomic Regulation (CRG): Juan Valcárcel, Elías Campo from IDIBAPS, and nurses from Occupational Health and Preventive Medicine departments at HCB who have contributed in several ways to make this study happen in the context of such high-pressure clinical care conditions. We are grateful to F. Krammer for donation of RBD protein, to Azucena Bardají for providing the nasopharyngeal swabs, Aida González (BETA Implants) for their fantastic face shields, Maria Jesús Mustieles for helping our team of nurses, to Leonie Mayer for assistance with literature review, to Gemma Ruiz-Olalla for statistical advice, to Javier Moreno for assistance with sample processing, to Jordi Vila and Mireia Navarro, for all the support at the Microbiology Department of HCB. Funding from this study comes from internal ISGlobal funds and in-kind contributions of HCB and CRG. GM had the support of the Department of Health, Catalan Government (SLT006/17/00109). Development of SARS-CoV-2 reagents was partially supported by the NIAID Centers of Excellence for Influenza Research and Surveillance (CEIRS) contract HHSN272201400008C. We acknowledge support from the Spanish Ministry of Science and Innovation through the "Centro de Excelencia Severo Ochoa 2019-2023" Program (CEX2018-000806-S), and support from the Generalitat de Catalunya through the CERCA Program.

\section{Author contributions}

A.L.G.B. and G.M. are joint first authors. M.T. and M.V. are joint second authors. C.D. and A.M. are joint senior authors. A.L.G.B., G.M., A.M., C.B., C.G. designed the study. E.C., A.C., S.F., M.L., N.O., N.P., M.R., N.R., P.S., S.T., S.W. recruited participants, collected data, obtained samples at the clinic. A.J., M.V., R.A., D.B., S.M., R.M., M.J.M. L.P., P.C., S.A., C.J. processed the samples, developed and performed the serological assays and analysis. A.M., C.C., P.C., J.H. conducted or supervised the molecular analysis (rRT-PCR). S.B., A.V., A.L., J.M., A.T., P.V. contributed to the critical interpretation of the results. C.C., R.S., S.M. and S.S. managed the data and performed the statistical analysis. A.L.G.B., G.M., A.M., C.D., C.G. wrote the first draft of the paper. All authors approved the final version as submitted to the journal.

\section{Competing interests}

The authors declare no competing interests.

\section{Additional information}

Supplementary information is available for this paper at https://doi.org/10.1038/s41467020-17318-x.

Correspondence and requests for materials should be addressed to A.L.G.-B.

Peer review information Nature Communications thanks the anonymous reviewers for their contribution to the peer review of this work.

Reprints and permission information is available at http://www.nature.com/reprints

Publisher's note Springer Nature remains neutral with regard to jurisdictional claims in published maps and institutional affiliations.

(c) (i) Open Access This article is licensed under a Creative Commons Attribution 4.0 International License, which permits use, sharing, adaptation, distribution and reproduction in any medium or format, as long as you give appropriate credit to the original author(s) and the source, provide a link to the Creative Commons license, and indicate if changes were made. The images or other third party material in this article are included in the article's Creative Commons license, unless indicated otherwise in a credit line to the material. If material is not included in the article's Creative Commons license and your intended use is not permitted by statutory regulation or exceeds the permitted use, you will need to obtain permission directly from the copyright holder. To view a copy of this license, visit http://creativecommons.org/ licenses/by/4.0/.

(C) The Author(s) 2020 